\title{
NOTE: A Versatile Mass Spectrometer Chamber for Molecular Beam and Temperature Programmed Desorption Experiments
}

\author{
James P. Tonks, ${ }^{1,2, a)}$ Ewan C. Galloway, ${ }^{2,}$ b) Martin O. King, ${ }^{2}$ Gwilherm Kerherve, ${ }^{3, c)}$ and John F. Watts ${ }^{1}$ \\ ${ }^{1)}$ Department of Mechanical Engineering Sciences, University of Surrey, Guildford, Surrey, GU2 $7 X H$, \\ $U K$ \\ 2) AWE plc, Aldermaston, Reading, Berkshire, RG7 4PR, UK \\ ${ }^{3)}$ VACGEN ${ }^{T M}$ Ltd, St. Leonards-on-Sea, East Sussex, TN38 9NN, UK
}

(Dated: 7 July 2016)

A dual purpose mass spectrometer chamber capable of performing molecular beam scattering (MBS) and temperature programmed desorption (TPD) is detailed. Two simple features of this design allow it to perform these techniques. Firstly, the diameter of entrance aperture to the mass spectrometer can be varied to maximize signal for TPD or to maximize angular resolution for MBS. Secondly, the mass spectrometer chamber can be radially translated so that it can be positioned close to the sample to maximize signal or far from the sample to maximize angular resolution. The performance of this system is described and compares well with systems designed for only one of these techniques. (C) British Crown Owned Copyright $\mathrm{AWE} / 2016$

Molecular beam scattering (MBS) and temperature programmed desorption (TPD) are complementary techniques that have been widely applied to study interactions at the solid-gas interface. Both techniques have played a leading role in determining adsorption dynamics; ${ }^{1-3}$ surface structure and physics; ${ }^{4-6}$ and reaction kinetics. ${ }^{7-9}$ However, the experimental geometry demanded by MBS and TPD are entirely different. To provide increased time and angular resolution, MBS experiments are performed with the mass spectrometer positioned typically $10 \mathrm{~s}$ of $\mathrm{cm}$ from the sample, with a narrow entrance aperture. ${ }^{10-12}$ Conversely, TPD requires the signal to be maximized, therefore a short sample detector distance, typically $1 \mathrm{~mm}$, with a large entrance aperture is required. ${ }^{13}$ Although TPD and MBS have been previously reported with the same detector, it has been at the expense of resolution. ${ }^{14,15}$ This has led to systems being designed either for MBS or TPD or having separate chambers for TPD and MBS. This note describes an instrument that allows TPD and high resolution MBS to be performed with the same mass spectrometer, in which the spectrometer can be moved along both the polar and radial axes and the width of the entrance aperture varied.

The system comprises five separate ultra high vacuum (UHV) chambers. There is a sample preparation chamber with standard cleaning facilities $\mathrm{Ar}^{+}$sputtering, annealing), gas dosing and metal deposition. An analytical chamber is also present, which has the capability for x-ray photoelectron spectroscopy (XPS), ultraviolet photoelectron spectroscopy (UPS), Auger electron spectroscopy (AES) and scanning electron microscopy (SEM). The scattering, mass spectrometer and molecular beam forming chambers are described below.

The molecular beam is generated through free-jet expansion of a high pressure gas through a converging nozzle into a low pressure background. The system described

\footnotetext{
a) james.tonks@awe.co.uk

b) ewan.galloway@awe.co.uk

c) Current Address: Department of Materials, Imperial College London, London, SW7 2BP, UK
}

here uses a Gottingen type design, which utilizes a relatively low pressure within the nozzle with a faster pumping speed to provide the pressure difference. This type of source has been comprehensively described by many different authors. ${ }^{11,12,16-19}$ The molecular beam used here features a $50 \mu \mathrm{m}$ quartz nozzle pressurized between 4 and 5 bar (typically 4.5 bar), with expansion and modulation stages pumped by 4600 and $3600 \mathrm{l} \mathrm{s}^{-1}$ baffled oil diffusion pumps, respectively. The base pressure of these chambers is $10^{-8}$ mbar. The beam is extracted by a $500 \mu \mathrm{m}$ skimmer, situated between the expansion and modulation chambers. Separation between the nozzle and skimmer was adjusted to optimise beam intensity. During use, the pressure in the expansion chamber increases from $10^{-8}$ to $10^{-4}$ mbar at He flows of typically $2.5-3.3 \mathrm{mbar} \mathrm{s}^{-1}$. The beam is mechanically chopped by a rotating disk to provide a pulsed beam of frequency up to $1000 \mathrm{~Hz}$. The chopper has two different sized slits allowing for the beam to chopped into $\sim 1.5 \%$ or $50 \%$ pulses. The time of flight (ToF) of the beam is measured by a LED and a photodiode system, whereby a timing signal is sent to the mass spectrometer to begin detection. A time channel width of $0.1 \mu \mathrm{s}$ was used. There is also a pneumatically driven shutter that allows the beam to be turned on or off in under $100 \mu \mathrm{s}$. A variety of collimating apertures are installed $(4,2,1.5,1,0.5$ and $0.2 \mathrm{~mm})$, allowing for the size of the beam to be changed. The nozzle can be heated up to $1000 \mathrm{~K}$, via resistive heating.

The $635 \mathrm{~mm}$ diameter scattering chamber achieves a base pressure of $2 \times 10^{-10}$ mbar through a combination of a $1100 \mathrm{ls}^{-1}$ turbomolecular pump and a titanium sublimation pump. The sample manipulator in this chamber has 6 degrees of freedom $(x, y, z$ and rotations in polar and azimuthal directions) and has a temperature range of $180-1000 \mathrm{~K}$. The quadrupole mass spectrometer (HIDEN HAL 7, 0-200 amu range) is housed in a separate, differentially pumped, chamber. Figure 1 shows a photograph of the mass spectrometer chamber, housed within the larger scattering chamber. The mass spectrometer chamber is mounted on a goniometer which is sealed from the scattering chamber with a PTFE ring. The rotational 
range of the mass spectrometer is $\sim 200^{\circ}$, driven by an externally mounted stepper motor with a step resolution of $0.01^{\circ}$. Radial motion of the chamber is enabled by a linear drive and a bellow, providing about $240 \mathrm{~mm}$ of travel. The linear drive is operated by a stepper motor driven screwdriver with a step increment of $1 \mu \mathrm{m}$. The mass spectrometer housing contains different sized apertures $(5,3,2$ and $1 \mathrm{~mm}$ diameter) selectable by rotating the housing using a screwdriver mounted to a feedthrough, thereby allowing the aperture size to be selected for the experiment. To reduce the background signal in the mass spectrometer chamber an additional $1300 \mathrm{ls}^{-1}$ magnetically levitated turbomolecular pump backed by a $701 \mathrm{~s}^{-1}$ turbomolecular pump and a titanium sublimation pump are fitted. The maximum and minimum distances between sample and mass spectrometer are 237 and $1 \mathrm{~mm}$, respectively. Using a $237 \mathrm{~mm}$ sample-detector distance and a $1 \mathrm{~mm}$ entrance aperture, the angle subtended from the sample to detector is $\sim 0.24^{\circ}$. The selectable aperture diameter and linear motion are the two key design features allowing this mass spectrometer to perform TPD, He atom scattering (HAS) and MBS experiments.

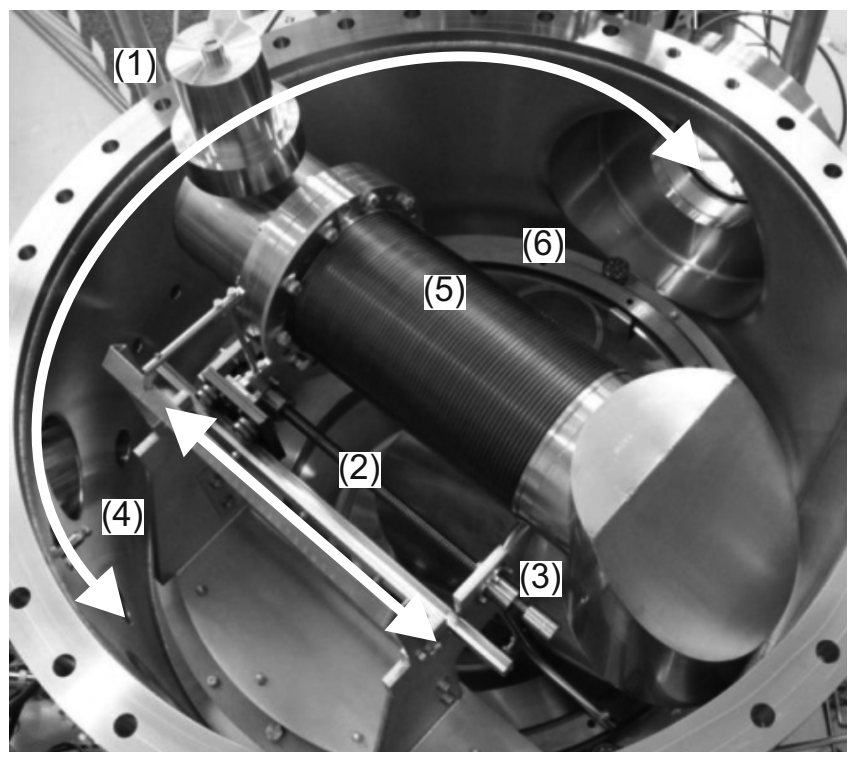

FIG. 1. Labelled photo of the mass spectrometer chamber. Arrows show the axes of motion; (1) rotatable mass spectrometer housing; (2) linear drive; (3) linear drive socket; (4) linear drive screwdriver; (5) bellow; (6) goniometer.

The drift velocity $\left(v_{d}\right)$ of the He beam was measured by noting the arrival time with the mass spectrometer positioned at 0 and $180^{\circ}$ in the scattering chamber. For a nozzle temperature of $298 \mathrm{~K}(\mathrm{E}=64 \mathrm{meV}), v_{d}$ is calculated as $1758 \mathrm{~m} \mathrm{~s}^{-1}$, in agreement with theory. ${ }^{20}$ The monochromaticity of the beam was determined from the width of the He pulse. The obtained ToF signal was fitted to the convolution of mathematical description of the gate function and the velocity distribution through least squares fitting. The fit gives a velocity spread $(\Delta v / v)$ of $8.4 \pm 0.6 \%$, corresponding to a beam temperature $\left(T_{B}\right)$ of $2.8 \pm 0.1 \mathrm{~K}$ and a Mach number of $19 \pm 1$, similar to other sources of this type. ${ }^{11,12,16-19}$ The beam number density was measured by calibrating the response of the mass spectrometer to a spinning rotor gauge. A high molecular beam flux is desirable as scattered molecular beams are typically of the order of a few percent of the incident beam flux. ${ }^{5}$ The intensity of a beam can be defined as the number density, the number of atoms per unit volume, $N_{v}$. Using a collimating aperture of $1 \mathrm{~mm}$ diameter and a He beam stagnation pressure of 4.5 bar, a pressure of $8.4 \times 10^{-6} \mathrm{mbar}$ was measured on the calibrated mass spectrometer, which gives $N_{v}=2.04 \times 10^{11} \mathrm{~cm}^{-3}$. The He atom flux, $N_{f}$, is the number of atoms impinging on a unit area per unit time, which was calculated using $v_{d}$ to be $3.59 \times 10^{16} \mathrm{~cm}^{-2} \mathrm{~s}^{-1}$. Such a flux is of the order of 10 monolayer $\mathrm{s}^{-1}$, which is appropriate for reactive scattering studies; however it is at the upper end of the desired range and can be reduced through the use of a smaller collimating aperture or the $1.5 \%$ or $50 \%$ duty cycles on the chopper. The beam divergence was measured by rotating the mass spectrometer so that it crossed the beam. The divergence places a limit on the angular resolution achievable in an experiment. It was found that a $1 \mathrm{~mm}$ collimating aperture provided the best compromise between intensity and resolution. The angular resolution of the beam in this configuration is $1.44^{\circ}$.

A series of experiments were conducted to determine the experimental performance of this system. HAS was performed on an atomically clean $\mathrm{Ni}(100)$ surface at $295 \mathrm{~K}$ and is shown in figure 2a. The detector was placed at $237 \mathrm{~mm}$ from the sample with a $1 \mathrm{~mm}$ diameter entrance aperture. As $\mathrm{Ni}$ is a free electron metal, only the specular reflection was visible. The full width half maximum of the specular reflection of the He beam from $\mathrm{Ni}(100)$ surface was measured to be $1.91^{\circ}$. The increase in the beam width with respect to the intrinsic width is probably due to a Debye-Waller effect. ${ }^{21}$

A TPD profile was obtained for $\mathrm{H}_{2}$ adsorbed on $\mathrm{Ni}(100)$ with a detector sample distance of $1 \mathrm{~mm}$ and an entrance aperture diameter of $5 \mathrm{~mm}$. The sample was prepared by exposing $\mathrm{Ni}(100)$, cooled to $200 \mathrm{~K}$, to $10 \mathrm{~L}$ ( $1 \mathrm{~L}=10^{-6}$ torrs) of $\mathrm{H}_{2}$. A TPD was then performed from $250-450 \mathrm{~K}$ at a ramp rate of $0.5 \mathrm{~K} \mathrm{~s}^{-1}$. The result of this experiment is shown in figure $2 \mathrm{~b}$, a clear $\mathrm{H}_{2}$ peak is evident at $300 \mathrm{~K}^{22}$

In addition, reactive scattering was performed by exposing the $\mathrm{Ni}(100)$ surface at $295 \mathrm{~K}$ to a He beam containing $1 \% \mathrm{O}_{2}$. The detector was placed normal to the sample surface at a distance of $35 \mathrm{~mm}$, with an entrance aperture of $5 \mathrm{~mm}$. A initial sticking probability of 0.58 was measured in this arrangement, comparable to literature. ${ }^{23}$

The performance of a multi purpose mass spectrometer chamber has been described. A series of experiments verified the effective operation of the mass spectrometer. A sharp $\mathrm{Ni}(100)$ specular reflection was obtained for HAS; for TPD, $\mathrm{H}_{2}$ desorption was characterised; and for reactive scattering, the sticking probability of $\mathrm{O}_{2}$ was obtained. All experiments compare well with literature.

\section{ACKNOWLEDGMENTS}

This work was supported by the EPSRC (Grant No: $\mathrm{EP} / \mathrm{G} 037388 / 1)$ and AWE plc as part of an EngD in 
(a)

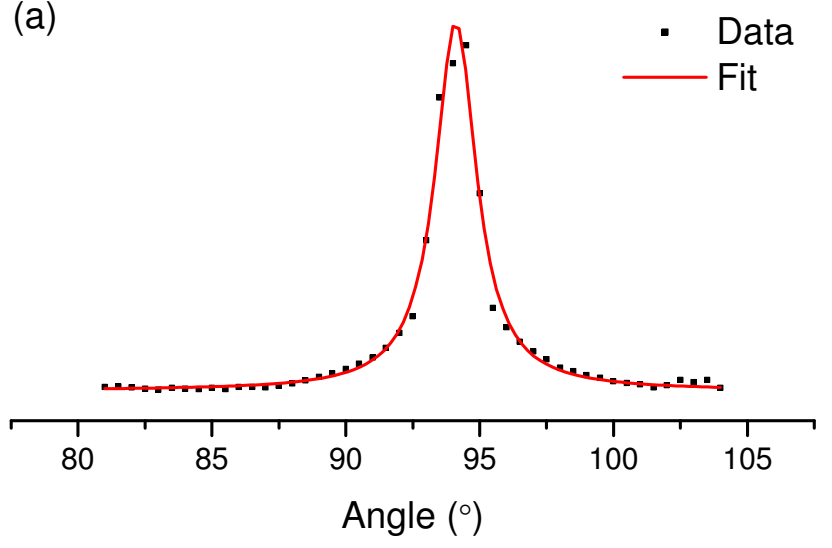

(b)

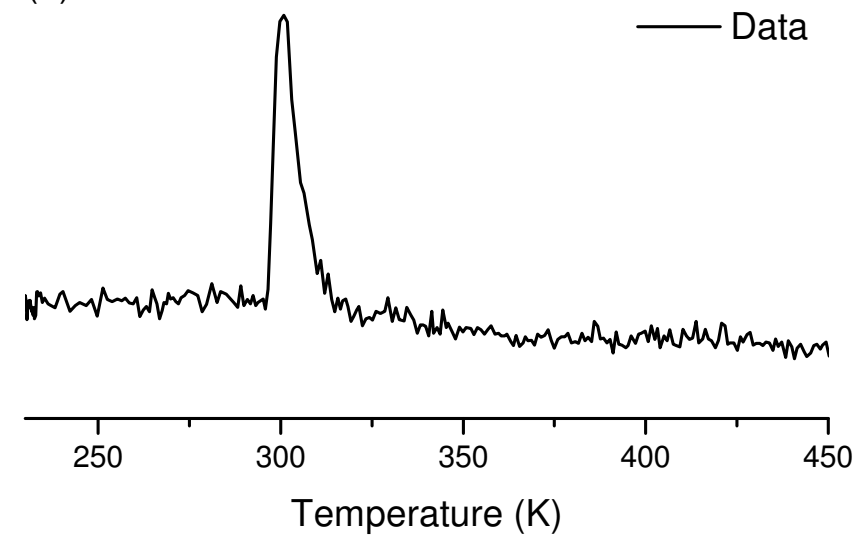

FIG. 2. (a) Specular reflection of a He beam from a Ni(100) surface using an incidence angle of $47^{\circ}$. The black dots show the raw data and the red line shows the fitted Lorentz distribution. (b) TPD trace of $m / z=2$ from $\mathrm{H}_{2}$ adsorbed on $\mathrm{Ni}(100)$ at $200 \mathrm{~K}$ using a ramp rate of $0.5 \mathrm{Ks}^{-1}$. the Centre for Doctoral Training in Micro and NanoMaterials and Technology at the University of Surrey. Special thanks to Scott Lawrence and David Bentley of VACGEN Ltd.

${ }^{1}$ M. A. Henderson, Surf. Sci. Rep. 46, 1 (2002).

${ }^{2}$ J. Wang and U. Burghaus, J. Chem. Phys. 122, 044705 (2005).

${ }^{3}$ B. Poelsema, K. Lenz, and G. Comsa, J. Phys.: Condens. Matter 22, 304006 (2010).

${ }^{4}$ G. Brusdeylins, R. B. Doak, and J. P. Toennies, Phys. Rev. Lett. 44, 1417 (1980).

${ }^{5}$ D. Farias and K. H. Rieder, Rep. Prog. Phys. 61, 1575 (1998).

${ }^{6}$ A. P. Graham, Surf. Sci. Rep. 49, 115 (2003).

${ }^{7}$ M. P. D'Evelyn and R. J. Madix, Surf. Sci. Rep. 3, 413 (1984).

${ }^{8}$ C. R. Arumainayagam and R. J. Madix, Prog. Surf. Sci. 38, 1 (1991).

${ }^{9}$ M. Okada, J. Phys.: Condens. Matter 22, 263003 (2010).

${ }^{10}$ A. Tamtogl, M. Mayrhofer-Reinhartshuber, N. Balak, W. Ernst, and K. Rieder, Journal of Physics: Condensed Matter 22, 304019 (2010).

${ }^{11}$ M. J. Cardillo, C. S. Y. Ching, E. F. Greene, and G. E. Becker, J. Vac. Sci. Technol. 15, 423 (1978).

${ }^{12}$ P. Nieto, D. Barredo, D. Farías, and R. Miranda, J. Chem. Phys. 115, 7283 (2011).

${ }^{13}$ D. P. Engelhart, R. J. V. Wagner, A. Meling, A. M. Wodtke, and T. Schfer, Surf. Sci, 650, 11 (2016).

${ }^{14}$ C. T. Campbell, G. Ertl, H. Kuipers, and S. Segner, Surf. Sci. 107, 220 (1981).

${ }^{15}$ M. F. Luo, D. A. MacLaren, I. G. Shuttleworth, and W. Allison, Chem. Phys. Lett. 381, 654 (2003).

${ }^{16}$ J. Lapujoulade and Y. Lejay, J. Chem. Phys. 63, 1389 (1975).

${ }^{17}$ G. Boato, P. Cantini, and L. Mattera, Surf. Sci. 55, 141 (1976).

${ }^{18}$ K. H. Rieder and T. Engel, Nucl. Instrum. Methods 170, 483 (1980).

${ }^{19}$ S. T. Ceyer, W. J. Siekhaus, and G. A. Somorjai, J. Vac. Sci. Technol. 19, 726 (1981).

${ }^{20}$ D. R. Miller, in Atomic and Molecular Beam Methods, Vol. 1, edited by G. Scoles (Oxford University Press, 1988) Chap. 2, p. 14 .

${ }^{21}$ G. Boato, in Atomic and Molecular Beam Methods, Vol. 2, edited by G. Scoles (Oxford University Press, 1988) Chap. 12, p. 341.

${ }^{22}$ K. Christmann, O. Schober, G. Ertl, and M. Neumann, J. Chem. Phys. 60, 4528 (1974).

${ }^{23}$ J. T. Stuckless, C. E. Wartnaby, N. Al-Sarraf, S. J. B. DixonWarren, K. M., and K. D. A., J. Chem. Phys. 106, 2012 (1997). 